\title{
Phosphorylation of REPS1 at Ser709 by RSK attenuates the recycling of transferrin receptor
}

\author{
Seong Heon Kim ${ }^{1,2}$, Jin-hwa Cho ${ }^{2}$, Bi-Oh Park ${ }^{2,3}$, Byoung Chul Park ${ }^{1,4}$, Jeong-Hoon Kim ${ }^{1,2, *}$, Sung Goo Park ${ }^{1,2, *} \mathcal{E}$ \\ Sunhong Kim ${ }^{2,5, \dagger}$ \\ ${ }^{1}$ Department of Functional Genomics, KRIBB School of Biological Science, Korea University of Science and Technology, Daejeon 34113, \\ ${ }^{2}$ Disease Target Structure Research Center, Korea Research Institute of Bioscience and Biotechnology, Daejeon 34141 , ${ }^{3}$ College of \\ Pharmacy, Chungbuk National University, Cheongju 28160, ${ }^{4}$ Department of Proteome Structural Biology, KRIBB School of Biological \\ Science, Korea University of Science and Technology, Daejeon 34113, ${ }^{5}$ Department of Biomolecular Science, KRIBB School of Biological \\ Science, Korea University of Science and Technology, Daejeon 34113, Korea
}

\begin{abstract}
RalBP1 associated EPS domain containing 1 (REPS1) is consenved from Drosophila to humans and implicated in the endocytic system. However, an exact role of REPS1 remains largely unknown. Here, we demonstrated that mitogen activated protein kinase kinase (MEK)-p90 ribosomal S6 Kinase (RSK) signaling pathway directly phosphorylated REPS1 at Ser709 upon stimulation by epidermal growth factor (EGF) and amino acid. While REPS2 is known to be involved in the endocytosis of EGF receptor (EGFR), REPS1 knockout (KO) cells did not show any defect in the endocytosis of EGFR. However, in the REPS1 KO cells and the KO cells reconstituted with a non-phosphorylatable REPS1 (REPS1 S709A), the recycling of transferrin receptor (TfR) was attenuated compared to the cells reconstituted with wild type REPS1. Collectively, we suggested that the phosphorylation of REPS1 at S709 by RSK may have a role of the trafficking of TfR. [BMB Reports 2021; 54(5): 272-277]
\end{abstract}

\section{INTRODUCTION}

Receptor tyrosine kinases, such as EGF receptor (EGFR) undergo the rapid internalization and degradation after the binding of their cognate ligands (1-5). In contrast, the transferrin (Tf) receptor (TfR), which binds di-ferric Tf to deliver iron into the cell,

*Corresponding authors. Sunhong Kim, Tel: +82-2-6987-5505; Fax: +82-2-6987-4844; E-mail: skimworm@gmail.com; Jeong-Hoon Kim, Tel: +82-42-860-4264; Fax: +82-42-860-4598; E-mail: jhoonkim@ kribb.re.kr; Sung Goo Park, Tel: +82-42-860-4262; Fax: +82-42-8604598; E-mail: sgpark@kribb.re.kr

${ }^{\dagger}$ Current affiliation: Drug Discovery Center, Life Sciences, LG Chem, Seoul 07796, Korea

https://doi.org/10.5483/BMBRep.2021.54.5.266

Received 2 December 2020, Revised 10 December 2020, Accepted 24 December 2020

Keywords: Attenuation, Phosphorylation, Recycling, RSK, Transferrin receptor internalizes constitutively and is recycled back to the plasma membrane (6-8). Both of the receptors utilize the clathrin coated pit for the internalization and subsequent endocytic machinery. The exact molecular mechanisms that confer the differences in the itinerary of the endocytosed EGFR and the TfR are, however, not entirely clear.

RalBP1 associated EPS domain containing 1 and 2 (REPS1 and REPS2) are first known to be associated with endocytosis and conserved from fly to human. REPS1 and REPS2 consist of 796 amino acids (87 kDa) and 660 amino acids (71 kDa), respectively. They all have two Eps homology (EH) domain, EFhand domain, and RalBP1 interaction site with high homology (9). REPS2, a much more studied one among two paralogues, has been implicated in endocytosis (10-12). It has been demonstrated that Reps2 regulates internalization of receptors, such as the EGFR via AP-2 complex of clathrin-coated vesicles, attenuating the receptor activities (13-16). In case of REPS1, it has been shown that Intersectin 1, one of the components of AP-2 complex, binds to REPS1 (17). However, its role in the receptor trafficking remains unclear.

Recently, a company have generated an antibody against phospho-S709-REPS1. The sequence around S709 took our attention because it was RXRXXS/T motif that was the consensus phosphorylation motif of AGC kinases, such as Akt, S6K1, and p90 ribosomal S6 kinase (RSK) $(18,19)$. Using various inhibitors for those kinases, we found out that RSK was a major kinase that phosphorylated REPS1 at S709. We showed that RSK directly binds and phosphorylates REPS1 via co-immunoprecipitation and in vitro kinase assay, and knockdown of RSK diminished the phosphorylation of REPS1 at S709. When REPS1 knockout (KO) cells were reconstituted by REPS1 wild type (WT) and Ser709Ala (SA), REPS1 KO and SA-expressing cells showed a delayed recycling of TfR compared to REPS1 WT-expressing cells, while the recycling of EGFR was not altered. Collectively, these results suggest that REPS1 may have a role in the recycling of TfR and the phosphorylation of REPS1 at S709 is required for the recycling. 


\section{RESULTS}

\section{The phosphorylation of REPS1 S709 was downregulated by MEK and RSK inhibitors}

Recently, cell signaling technology has developed an anti-phospho S709 REPS1 (p-REPS1) antibody, which detects the phosphorylation of S709, a consensus phosphorylation site by AGC kinase family. To assess the selectivity of the antibody, 293T cells and REPS1-KO HEK293E cells (Supplementary Fig. 1A) were transfected with Myc-tagged WT and SA REPS1 constructs. Anti-pREPS1 antibody detected endogenous and exogenous REPS1 WT, but not SA mutant (Fig. 1A). As mentioned earlier, S709 is a part of the conserved consensus phosphorylation motif for AGC kinase family. The kinase group AGC is one of the protein kinase family including protein kinase $A$ (PKA), G (PKG), $\mathrm{C}$ (PKC), Akt, and ribosomal protein S6 kinase (RSK). Many of those, such as Akt and PKC, are phosphorylated and activated by mTORC2 (20) and p70 S6 kinase is phosphorylated by mTORC1 (21). Only RSK is phosphorylated by its C-terminal kinase (22). S709 is a part of RXRXXS motif, which may be recognized and phosphorylated by Akt, RSK, and p70S6K, so we tested which kinase is responsible for the phosphorylation of REPS1 at S709. GDC0349 (mTORC1 and mTORC2 inhibitor), rapamycin (mTORC1 inhibitor), BI-D1870 (RSK inhibitor), and U0126 (MEK inhibitor), were treated to the cells and the cell lysates were analyzed by western blotting with anti-p-REPS1 antibody and others. In HEK293E cells, BI-D1870 significantly inhibited the phosphorylation of REPS1 at S709 (Fig. 1B, C). In addition, U0126 have shown the similar effect on REPS1 (Fig. 1B, C), implicating that MEK-ERK-RSK signaling may be involved in the phosphorylation at this site.

\section{Epidermal growth factor (EGF) or amino acid-induced phosphorylation of REPS1 was reduced by MEK and RSK inhibitors}

The previous study showed that REPS1 is tyrosine-phosphorylated in response to EGF stimulation (23). We wondered whether the phosphorylation at S709 is also regulated by EGF stimulation. EGF stimulation indeed elevated the phosphorylation of REPS1 at S709 significantly, but GDC0349 and rapamycin did not block this phosphorylation (Fig. 2A, B). However, BI-D1870 and U0126 almost abrogated the EGF-induced phosphorylation of REPS1 (Fig. 1A, B). The same phenomena were observed in HeLa cells and Caco-2 cells (Supplementary Fig. 2). These data indicated that EGF-induced Ras-MAPK-RSK signaling may elicit the phosphorylation of REPS1.

Next, we tested whether amino acid stimulation, which is known to activate mTORC1-p70S6K signaling, regulates the REPS1 phosphorylation. It was intriguing that amino acid stimulation to HEK293E cells led to the increase in the phosphorylation of REPS1 at S709 (Fig. 2C, D). The mTOR inhibitors (GDC0349 and rapamycin) and U0126 restored the level of phospho-S709 to the control, while BI-D1870 strongly reduced it below the control level (Fig. 2C, D). This result demonstrated that mTOR-
A

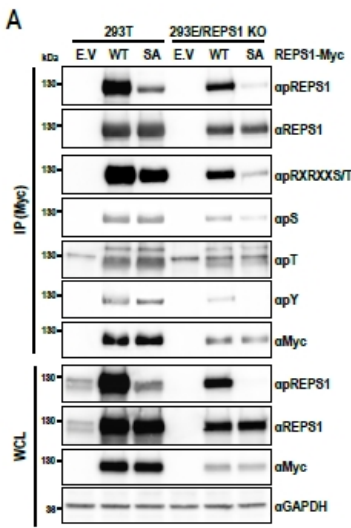

B

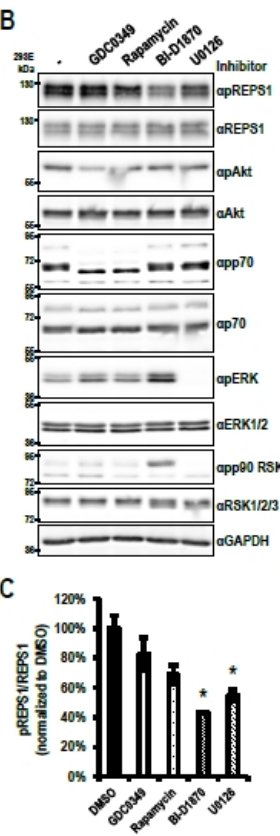

Fig. 1. Phosphorylation of REPS1 at S709 was controlled by MEK-RSK signaling. (A) 293T and 293E/REPS1 KO cell lysates transfected with REPS1-Myc constructs were subjected to immunoprecipitation with anti-Myc magnetic beads. Immunoblots were completed with the indicated antibodies. B-E. 293E (B, C) cells were treated with inhibitors, $1 \mu \mathrm{M}$ GDC0349, $1 \mu \mathrm{M}$ rapamycin, $10 \mu \mathrm{M}$ BI-D1870, $10 \mu \mathrm{M}$ U0126, for $30 \mathrm{~min}$. The intensity of the p-REPS1 bands was quantified by ImageJ program and divided by total REPS1. All the data were analyzed from two biological experiments to calculate a significance score using Student's t-test. $* \mathrm{P}<0.05$.

p70S6K signaling pathway can modulate the phosphorylation of REPS1 and, however, RSK is still the major kinase responsible for the phosphorylation. Taken together, we suggested that the phosphorylation of REPS1 at S709 is mediated by RSK.

RSK directly phosphorylates REPS1 at S709 in vitro Judging from the above data, the phosphorylation of REPS1 at 
A

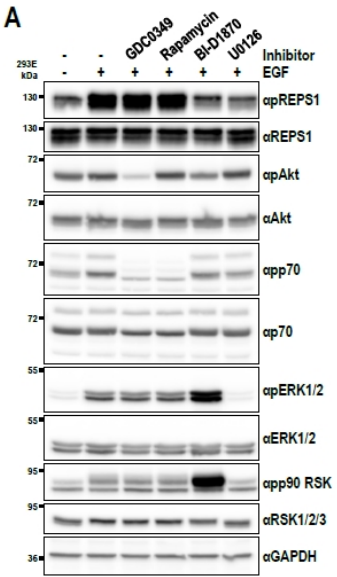

B

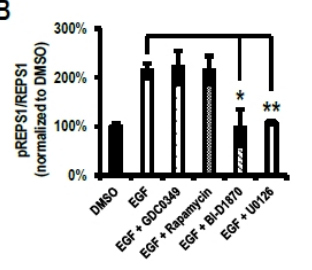

C

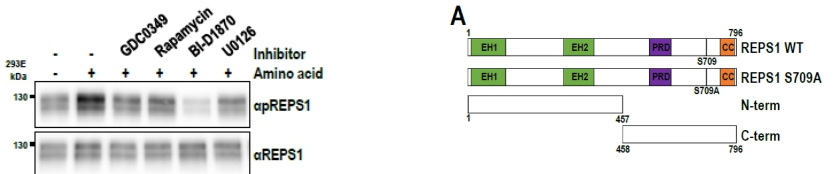

B

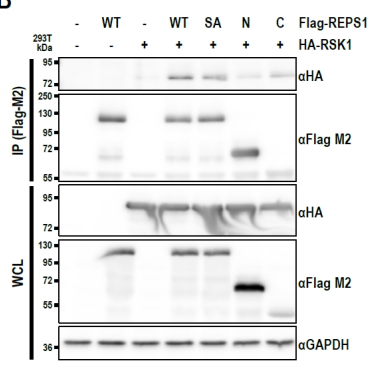

$C_{2}$

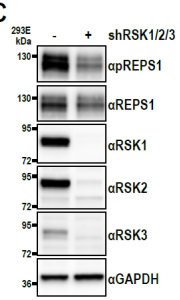

D

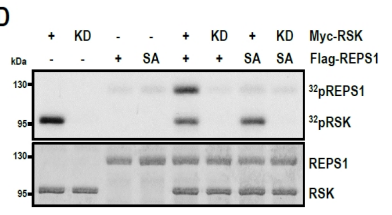

Fig. 3. RSK interacts with REPS1 and phosphorylates p-REPS1 S709. (A) The domain structure of REPS1 and its mutants. (B) 293T cells transfected with Flag-REPS1 and HA-RSK1 plasmids were subjected to co-immunoprecipitation with anti-Flag magnetic beads and the western blot were completed with anti-HA and anti-Flag M2 antibodies. (C) HEK239E cells stably expressing RSK1/2/3 shRNA were lysed and the lysates were subjected to immunoblots with the indicated antibodies. (D) In vitro kinase assay was carried out as described in Materials and Methods. The data presented here was a representative of two independent experiments.

wild type, and constitutively active (CA) and kinase dead (KD) mutants. Endogenous REPS1 S709 phosphorylation increased in RSK1 wild type and CA-transfected cells, but not in KD-expressed cells (Supplementary Fig. 3B, C). Confirming the direct phosphorylation further, we performed in vitro kinase assay using Myctagged chicken RSK and Flag-tagged REPS1. RSK wild type robustly phosphorylated REPS1 WT, but RSK KD did not (Fig. 3D). In addition, REPS1 SA was not phosphorylated by RSK wild type at all (Fig. 3D). Taken together, these data demonstrated that RSK directly phosphorylates REPS1 at S709 in vitro and in vivo.

\section{The phosphorylation of REPS1 at S709 was likely to be required for the internalization of transferrin receptor} Given that REPS1 is tyrosine phosphorylated after EGF stimulation (23) and interacts with clathrin-coated pit complex $(17,23)$, we hypothesized that REPS1 and its phosphorylated form at S709 may be involved in the endocytosis of EGF receptor as its paralogue, REPS2, does $(12,13,16,25,26)$. We made REPS1-KO HeLa cell line using CRISPR/Cas9 technology as described in Materials and Methods, and then, established stable cell lines expressing empty vector, REPS1 WT, and SA in KO cells (Supplementary Fig. 1). Those cells transfected with EGFR-GFP were treated with Alexa-488-conjugated EGF and subject to confocal microscopy. When EGF was bound to EGFR, they internalized and accumulated in the cytoplasmic speckles with or without REPS1 (Fig. 4A). Therefore, we concluded that REPS1 is not related to the endocytosis of EGFR complex.

Recently a published article insisted that REPS1 mutation asso- 
A
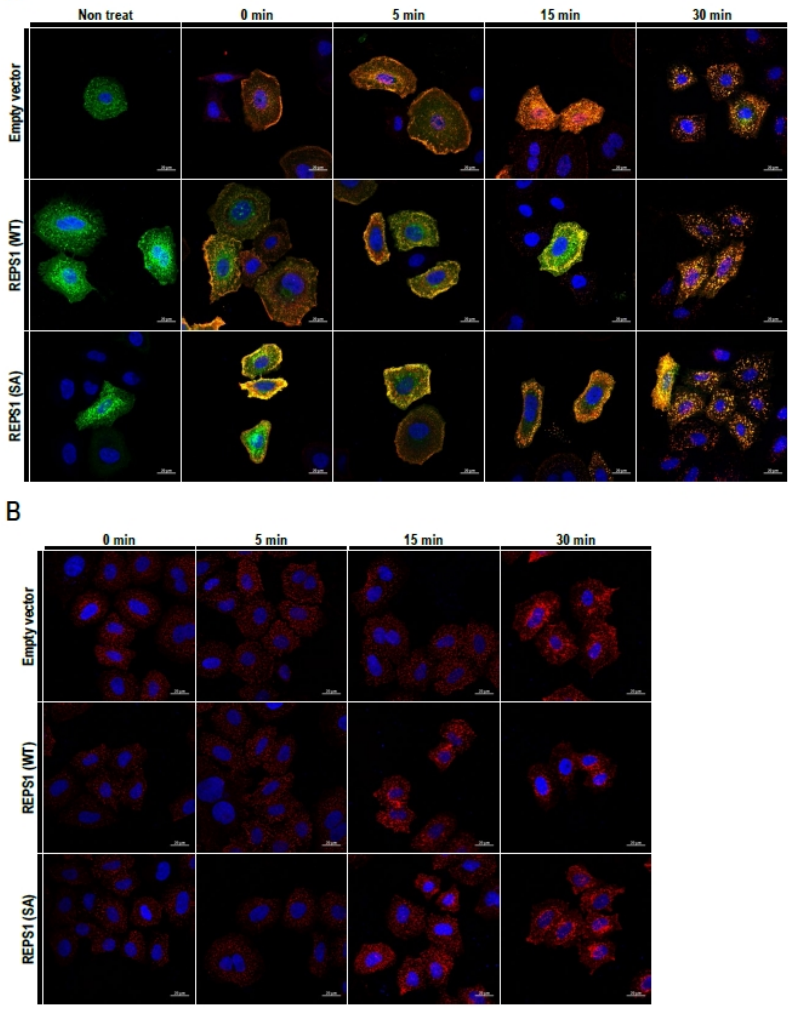

Fig. 4. The phosphorylation of REPS1 at S709 may be required for the recycling of TfR. (A) REPS1-KO HeLa cells expressing REPS1 wild type or SA mutant were transfected with EGFR-EGFP construct (green). $10 \mathrm{ng} / \mathrm{ml}$ Alexa-555 EGF (red) were added to the cells and they were visualized by confocal microscopy as described in Materials and Methods. (B) The same cell used in A were treated with $25 \mu \mathrm{g} /$ $\mathrm{ml}$ Alexa-555 transferrin (red) and the samples were subjected to a confocal microscopy as described in Materials and Methods.

ciated with neurodegeneration with brain iron accumulation may cause the defect in the recycling of TfR (27). To investigate a role of the phosphorylation of REPS1 at S709 in TfR recycling, we observed a kinetics of translocation of TfR using Alexa-555conjugated transferrin (RTf) in the same HeLa cell lines we prepared above. After the addition of RTf, the internalization of RTf-TfR complexes occurred (Fig. 4B, 0 and 5 min panels) and they accumulated in the perinuclear region and disappeared (Fig. 4B, 15 and 30 min panels of REPS1 WT). However, in the cells expressing empty vector and REPS1 SA, the perinuclear accumulation of RTf was attenuated and significant amount of them remained until $30 \mathrm{~min}$ after transferrin treatment (Fig. 4B). These data suggested that REPS1 and its phosphorylation at $\mathrm{S} 709$ are required for the recycling of TfR.

\section{DISCUSSION}

In this study, we found out that RSK mediates the phosphorylation of REPS1 at S709 induced by EGF and amino acid stimulation, and showed an evidence that this phosphorylation is associated with the recycling TfR, not with the endocytosis of EGFR. To our knowledge, it was the first evidence that the phosphorylation of REPS1 has a role in its physiological function.

REPS1 has been demonstrated to be associated with several clathrin-coated pit complex proteins and signaling adaptor proteins via its two $\mathrm{EH}$ domains and proline-rich motif $(13,15,16$, $25,28)$. However, its physiological roles were less studied compared to its closest isoform, REPS2. REPS2 has been demonstrated to be involved in the endocytosis of various receptors, such as EGFR (14) and Insulin receptor (26). It was intriguing that the endocytosis of transferrin receptor was not affected by REPS2 deletion mutant (26). We initially hypothesized that REPS1 may have a similar role with REPS2 because they share almost the same domain structure. However, we clearly demonstrated that the endocytosis of EGFR was unaffected in REPS1 KO and overexpressed cells (Fig. 4A). It seems that it also did not modulate the endocytosis of transferrin receptor like REPS2. We were surprised at the fact that REPS1 KO and SA mutant attenuated the recycling of the receptor (Fig. 4B). These results were consistent with the recent observations in which the mutations of REPS1 found in the patients with brain iron accumulation (NBIA), such as Val78Leu and Ala113Glu, may have caused the delayed recycling of transferrin receptor (27). Although the machinery that mediates the recycling of Tf/TfR complex is not well characterized, a couple of Rab family GTPases and dynamin have been known to be involved (29). There was also a report that Tf/TfR-containing endosomes generate buds in a clathrin-coat dependent manner $(30,31)$. Since it has been suggested that REPS1 is associated with clathrin-coat protein complex, REPS1 may be involved in this clathrin-dependent budding process of endosomes to be recycled. In sum, we suggest that REPS1 and its phosphorylation by RSK have a distinct role in vesicle trafficking.

It is of note that the S709 phosphorylation by RSK was induced in response to the treatments of EGF and amino acids. It is well known that EGF activates Ras-ERK-RSK signaling pathway, so it is conceivable that EGF can elicit the S709 phosphorylation. However, there is no evidence that amino acid supplementation can induce RSK activity. Thus, amino acid-mediated RSK activation should be further investigated elsewhere.

In conclusion, the phosphorylation of REPS1 at S709 by growth factor-induced RSK may be required for the recycling of transferrin receptor. The differential role of REPS1 and REPS2 in the context of endocytosis and recycling of membrane receptors should be investigated in the future. 


\section{MATERIALS AND METHODS}

\section{Cell culture and plasmid construction}

HEK293E, HEK293T, HeLa, and Caco-2 cells were maintained in Dulbecco's Modified Eagle Medium (Welgene, Gyeongsan, South Korea) supplemented with $10 \%$ fetal bovine serum (FBS), 1X Pen strep (Gibco, Tulsa, USA), and 1X glutamax (Gibco). pc DNA3-3xFlag-REPS1, pcDNA3.1-REPS1-myc His(-), and pcDNA3. 1-HA-RSK1 constructs were cloned. EGFR-EGFP plasmid was purchased from Addgene (32751, Watertown, MA, USA).

\section{Establishing REPS1 KO in 293E and HeLa cell lines}

Knockout target sequences are as follows: REPS1 KO: 5'-CC AAGAAGGTGGTGGTCAAC-3' (for 293E cell lines), 5'-AGAAGG TGGTGGTCAACGGG-3' (for HeLa cell lines). REPS1 knockout cell lines were established by pL-CRISPR.SFFV.tRFP vector (57826, Addgene). For REPS1-reconstituted cell lines, REPS1 (WT and SA)Myc were cloned into pLVX-EF1 $\alpha$-IRESpuro vector and the cells were infected with the lentivirus from the construct and selected with $1 \mu \mathrm{g} / \mathrm{ml}$ puromycin.

\section{Antibodies and reagents}

Primary antibodies used in this study were as follows: anti-phospho REPS1 S709 (12005), anti-REPS1 (6404), anti-phospho Akt S473 (4060), anti-Akt (4691), anti-phospho p70 S6K (9234), antip70 S6K (2708), anti-phospho threonine (9386), anti-phospho tyrosine (9416), anti-phospho ERK T202/T204 (4370), anti-ERK1/2 (4695), anti-phospho RSK S380 (11989), anti-RSK1/2/3 (9355), anti-RSK1 (8408), anti-RSK2 (5528), anti-Myc (2278) were purchased from Cell Signaling Technology (Danvers, MA, USA). anti-phospho serine (61-8100, Invitrogen, Carlsbad, CA, USA), anti-RSK3 (sc-1431, Santa Cruz, Dallas, Texas, USA), anti-GAPDH (LF-PA0212, AB frontier, Seoul, South Korea), anti-Flag-M2 (F1804, Sigma Aldrich, Kenilworth, NJ, USA), and anti-HA (11 867423 001, Roche, Basel, Switzerland). Flag magnetic beads (A36798) and Myc magnetic beads (88842) were purchased from Thermo Fisher (Waltham, MA, USA). GDC0349 (S8040, Selleckchem, Houston, TX, USA), rapamycin (37094, Sigma Aldrich, Kenilworth, NJ, USA), BI-D1870 (S2843, Selleckchem), and U0126 (1144, Selleckchem) were additionally used for experiments in this study. Alexa-555 EGF (E35350) and Alexa-555 Transferrin (T35352) were purchased from Thermo Fisher.

\section{Preparation of cell lysates and immunoprecipitation}

After cells were washed with cold PBS, they were lysed in lysis buffer A (20 mM Tris-HCl (pH 7.5), $100 \mathrm{mM} \mathrm{NaCl}, 1$ mM EDTA, 2 mM EGTA, 1\% Triton X-100, 1X phosphatase inhibitor (B15001, Bimake, Houston, TX, USA), 1X protease inhibitor (P3100, Gen DEPOT, Katy, TX, USA), 1 mM PMSF, 1 mM DTT. Each lysates for immunoprecipitation was mixed with the equal volume of lysis buffer and incubated with pre-washed magnetic beads for overnight at $4^{\circ} \mathrm{C}$ on a rotator. The beads were washed with lysis buffer A three times (Fig. 1A) or lysis buffer A twice, 500 $\mathrm{mM} \mathrm{NaCl}$ lysis buffer $\mathrm{A}$ twice, and then lysis buffer $\mathrm{A}$ once again (Fig. 3B).

\section{In vitro kinase assay}

HEK293T cells transfected with Flag-REPS1 wild-type, SA mutant, Myc-chicken RSK wild-type and kinase-dead (KD) mutant (K112R), respectively, were lysed using lysis buffer $B$ (50 mM HEPES $\mathrm{KOH} \mathrm{pH} 7.4,40 \mathrm{mM} \mathrm{NaCl}, 1 \mathrm{mM}$ EDTA, 1 mM EGTA, $10 \mathrm{mM}$ $\mathrm{Na}$ pyrophosphate, $10 \mathrm{mM} \mathrm{Na} \beta$-glycerophosphate, $50 \mathrm{mM}$ $\mathrm{NaF}, 1 \mathrm{mM} \mathrm{NaVO} 4,1 \%$ Triton $\mathrm{X}-100$ ) containing protease inhibitor. The clarified lysates were immunoprecipitated with antiFlag-magnetic beads (for Flag-REPS1) or anti-c-Myc-magnetic beads (for Myc-RSK) at $4^{\circ} \mathrm{C}$ for $3 \mathrm{~h}$. The beads were washed twice in lysis buffer $B$, twice in $500 \mathrm{mM} \mathrm{NaCl}$ lysis buffer $B$, once in lysis buffer $B$ and once in kinase buffer $(25 \mathrm{mM} \mathrm{HEPES}-\mathrm{KOH}$ pH7.4, $10 \mathrm{mM} \mathrm{MgCl}$, $3 \mathrm{mM} \beta$-mercaptoethanol, $0.1 \mathrm{mg} / \mathrm{ml}$ BSA, $1 \mathrm{mM}$ DTT). In the last washing step, Flag-REPS1 and Myc-RSK binding beads were combined as indicated. The kinase assay was performed by the method previously described (32).

\section{Immnunocytochemistry}

HeLa/REPS1 KO cells infected with REPS1 (WT or SA)-Myc were seeded on poly-l-lysine-coated $\mu$-slide 8 well plates (80826, ibidi, Grafelfing, Germany). After serum starvation overnight, the plates were put on $4^{\circ} \mathrm{C}$ for $10 \mathrm{~min}$. The cells were treated with $10 \mathrm{ng} / \mathrm{ml}$ Alexa-555 EGF or $25 \mu \mathrm{g} / \mathrm{ml}$ Alexa-555 transferrin at $4^{\circ} \mathrm{C}$ for $50 \mathrm{~min}$. Then, the cells were transferred to $37^{\circ} \mathrm{C}$ incubator for indicated times. The cells were fixed by $4 \%$ paraformaldehyde for $20 \mathrm{~min}$ at room temperature. Nucleus were stained by mounting solution with DAPI (ab104139, Abcam, Cambridge, UK) and Fluorescence was visualized using LSM 880 confocal microscope. Scale bar. $20 \mu \mathrm{m}$.

\section{Statistical analysis}

Statistical significance of differences was tested using Student's $t$ test in Microsoft Excel $\left({ }^{*} \mathrm{P}<0.05,{ }^{*} \mathrm{P}<0.01,{ }^{* * *} \mathrm{P}<0.005\right)$. Western blots were quantified using ImageJ. The error bars represent standard deviation.

\section{ACKNOWLEDGEMENTS}

This work was supported by a grant (CAP-15-11-KRICT) from the National Research Council of Science and Technology, Ministry of Science, ICT, and Future Planning, a grant (NRF-2019 M3E5D4069882) from the National Research Foundation, Ministry of Science and ICT and Future Planning, and a grant from the KRIBB Initiative Program.

\section{CONFLICTS OF INTEREST}

The authors have no conflicting interests. 


\section{REFERENCES}

1. Levkowitz G, Waterman H, Ettenberg SA et al (1999) Ubiquitin ligase activity and tyrosine phosphorylation underlie suppression of growth factor signaling by $\mathrm{c}-\mathrm{Cbl} / \mathrm{Sli}-1$. Mol Cell 4, 1029-1040

2. Ettenberg SA, Keane MM, Nau MM et al (1999) cbl-b inhibits epidermal growth factor receptor signaling. Oncogene 18, 1855-1866

3. Biscardi JS, Maa MC, Tice DA, Cox ME, Leu TH and Parsons SJ (1999) c-Src-mediated phosphorylation of the epidermal growth factor receptor on Tyr845 and Tyr1101 is associated with modulation of receptor function. J Biol Chem 274, 8335-8343

4. Emlet DR, Moscatello DK, Ludlow LB and Wong AJ (1997) Subsets of epidermal growth factor receptors during activation and endocytosis. J Biol Chem 272, 4079-4086

5. Rojas M, Yao S and Lin YZ (1996) Controlling epidermal growth factor (EGF)-stimulated Ras activation in intact cells by a cell-permeable peptide mimicking phosphorylated EGF receptor. J Biol Chem 271, 27456-27461

6. Luck AN and Mason AB (2012) Transferrin-mediated cellular iron delivery. Curr Top Membr 69, 3-35

7. Abdel Shakor AB, Atia MM, Kwiatkowska K and Sobota A (2012) Cell surface ceramide controls translocation of transferrin receptor to clathrin-coated pits. Cell Signal 24, 677-684

8. Harding C, Heuser J and Stahl P (1983) Receptor-mediated endocytosis of transferrin and recycling of the transferrin receptor in rat reticulocytes. J Cell Biol 97, 329-339

9. Xu J, Zhou Z, Zeng L et al (2001) Cloning, expression and characterization of a novel human REPS1 gene. Biochim Biophys Acta 1522, 118-121

10. Morinaka K, Koyama S, Nakashima S et al (1999) Epsin binds to the $\mathrm{EH}$ domain of POB1 and regulates receptormediated endocytosis. Oncogene 18, 5915-5922

11. Oshiro T, Koyama $S$, Sugiyama $S$ et al (2002) Interaction of POB1, a downstream molecule of small G protein Ral, with PAG2, a paxillin-binding protein, is involved in cell migration. J Biol Chem 277, 38618-38626

12. Oosterhoff JK, Penninkhof F, Brinkmann AO, Anton Grootegoed J and Blok LJ (2003) REPS2/POB1 is downregulated during human prostate cancer progression and inhibits growth factor signalling in prostate cancer cells. Oncogene 22, 2920-2925

13. Tomassi L, Costantini A, Corallino $S$ et al (2008) The central proline rich region of POB1/REPS2 plays a regulatory role in epidermal growth factor receptor endocytosis by binding to 14-3-3 and $\mathrm{SH} 3$ domain-containing proteins. BMC Biochem 9, 21

14. Burke P, Schooler K and Wiley HS (2001) Regulation of epidermal growth factor receptor signaling by endocytosis and intracellular trafficking. Mol Biol Cell 12, 1897-1910

15. Kariya K, Koyama S, Nakashima S, Oshiro T, Morinaka K and Kikuchi A (2000) Regulation of complex formation of POB1/epsin/adaptor protein complex 2 by mitotic phosphorylation. J Biol Chem 275, 18399-18406

16. Ikeda M, Ishida O, Hinoi T, Kishida S and Kikuchi A (1998) Identification and characterization of a novel protein interacting with Ral-binding protein 1, a putative effector pro- tein of Ral. J Biol Chem 273, 814-821

17. Dergai O, Novokhatska O, Dergai M et al (2010) Intersectin 1 forms complexes with SGIP1 and Reps1 in clathrin-coated pits. Biochem Biophys Res Commun 402, 408-413

18. Kannan N, Haste N, Taylor SS and Neuwald AF (2007) The hallmark of AGC kinase functional divergence is its C-terminal tail, a cis-acting regulatory module. Proc Natl Acad Sci U S A 104, 1272-1277

19. Moritz A, Li Y, Guo A et al (2010) Akt-RSK-S6 kinase signaling networks activated by oncogenic receptor tyrosine kinases. Sci Signal 3, ra64

20. Ikenoue T, Inoki K, Yang Q, Zhou X and Guan KL (2008) Essential function of TORC2 in PKC and Akt turn motif phosphorylation, maturation and signalling. EMBO J 27, 1919-1931

21. Ahmed AR, Owens RJ, Stubbs CD et al (2019) Direct imaging of the recruitment and phosphorylation of S6K1 in the mTORC1 pathway in living cells. Sci Rep 9, 3408

22. Dalby KN, Morrice N, Caudwell FB, Avruch J and Cohen $P$ (1998) Identification of regulatory phosphorylation sites in mitogen-activated protein kinase (MAPK)-activated protein kinase-1a/p90rsk that are inducible by MAPK. J Biol Chem 273, 1496-1505

23. Yamaguchi A, Urano T, Goi T and Feig LA (1997) An Eps homology $(\mathrm{EH})$ domain protein that binds to the Ral-GTPase target, RalBP1. J Biol Chem 272, 31230-31234

24. Dummler BA, Hauge C, Silber J et al (2005) Functional characterization of human RSK4, a new 90-kDa ribosomal S6 kinase, reveals constitutive activation in most cell types. J Biol Chem 280, 13304-13314

25. Oosterhoff JK, Kuhne LC, Grootegoed JA and Blok LJ (2005) EGF signalling in prostate cancer cell lines is inhibited by a high expression level of the endocytosis protein REPS2. Int J Cancer 113, 561-567

26. Nakashima S, Morinaka K, Koyama S et al (1999) Small G protein Ral and its downstream molecules regulate endocytosis of EGF and insulin receptors. EMBO J 18, 3629-3642

27. Drecourt A, Babdor J, Dussiot $M$ et al (2018) Impaired transferrin receptor palmitoylation and recycling in neurodegeneration with brain iron accumulation. Am J Hum Genet 102, 266-277

28. Penninkhof F, Grootegoed JA and Blok LJ (2004) Identification of REPS2 as a putative modulator of NF-kappaB activity in prostate cancer cells. Oncogene 23, 5607-5615

29. van Dam EM and Stoorvogel W (2002) Dynamin-dependent transferrin receptor recycling by endosome-derived clathrincoated vesicles. Mol Biol Cell 13, 169-182

30. Stoorvogel W, Oorschot V and Geuze HJ (1996) A novel class of clathrin-coated vesicles budding from endosomes. J Cell Biol 132, 21-33

31. Futter CE, Gibson A, Allchin EH et al (1998) In polarized MDCK cells basolateral vesicles arise from clathrin-gammaadaptin-coated domains on endosomal tubules. J Cell Biol 141, 611-623

32. Cho JH, Kim SA, Seo YS et al (2017) The p90 ribosomal S6 kinase-UBR5 pathway controls Toll-like receptor signaling via miRNA-induced translational inhibition of tumor necrosis factor receptor-associated factor 3. J Biol Chem 292, 1180411814 